\title{
Adipokines correlate with pain in lower limb osteoarthritis: different associations in hip and knee
}

\author{
Sylvette Bas • Axel Finckh • Gabor J. Puskas • \\ Domizio Suva • Pierre Hoffmeyer • Cem Gabay • \\ Anne Lübbeke
}

Received: 22 April 2014 / Accepted: 4 June 2014 /Published online: 9 July 2014

(C) SICOT aisbl 2014

\begin{abstract}
Purpose Our aim was to investigate whether serum and synovial-fluid (SF) concentrations of interleukin-6 (IL-6), leptin, adiponectin, resistin or visfatin are associated with joint pain in hip and knee in end-stage osteoarthritis (OA).

Methods A cross-sectional study assessing patients with hip and knee OA undergoing total joint arthroplasty between January and December 2010 was conducted at a large university hospital. Serum and SF cytokine and adipokine concentrations were determined in samples obtained on the day of surgery. The main outcome was pain severity measured pre-operatively using the Western Ontario and McMaster Universities Osteoarthritis Index (WOMAC) and visual analogue scale (VAS) pain scores.

Results A total of 206 patients were involved (112 with hip and 94 with knee OA). Median age was 72 years [interquartile range (IQR) 66-79], $59 \%$ were women. All adipokine levels were significantly higher in the SF of hip joints than in that of knee joints, except for leptin, which tended to be higher in the knee. In both hip and knee OA, median serum concentrations of leptin, adiponectin, resistin and visfatin exceeded those in SF, whereas for IL-6, median concentrations were much higher in SF than in serum. In hip OA, worse pain was significantly associated with high SF concentrations of IL-6, visfatin and leptin; in knee OA, it was associated with high SF leptin and low SF adiponectin concentrations and a low adiponectin-leptin ratio.
\end{abstract}

S. Bas $\cdot$ A. Finckh $\cdot$ C. Gabay

Division of Rheumatology, Geneva University Hospitals and

University of Geneva, Geneva, Switzerland

G. J. Puskas · D. Suva • P. Hoffmeyer • A. Lübbeke $(\triangle)$

Division of Orthopaedic Surgery, Geneva University Hospitals and University of Geneva, CH-1211 Geneva, Switzerland

e-mail: Anne.LubbekeWolff@hcuge.ch
Conclusion Our findings support a connection between intraarticular concentrations of several adipokines and severity of preoperative OA pain. However, the specific adipokines differed by joints: in hip OA, pain was associated with IL-6 and visfatin and in knee OA with adiponectin; leptin played a role in both hip and knee OA.

Keywords Adipokine - Osteoarthritis · Pain · Visfatin · Leptin

\section{Introduction}

Joint pain is the major clinical feature of osteoarthritis (OA), a disease caused by multiple and yet non completely understood pathways [23]. Adipokines, such as interleukin-6 (IL-6), leptin, adiponectin, resistin and visfatin, belong to the family of white-adipose-tissue-produced factors involved in modulating immune and inflammatory responses $[10,27]$ and are present in synovial fluid (SF) of patients with OA $[5,8,11,13,19$, 22]. Very few studies have investigated the relationship between these adipokines and patient-reported joint pain $[9,14$, 15]. Gandhi et al. reported that SF adiponectin-leptin ratio predicted pain in knee OA [9]. Massengale et al. [15] found that leptin serum concentration was associated with pain intensity in patients with chronic hand OA. Moreover, we recently found that leptin levels correlated with pain in endstage OA [14]. To our knowledge, no clinical study thus far has evaluated a possible relationship between other adipokines, such as visfatin and resistin, and hip or knee OA pain. Visfatin has proinflammatory and immunomodulating functions, including the increase of tumour necrosis factoralpha (TNF- $\alpha$ ), IL-1 $\beta$, IL- 6 and monocyte chemoattractant protein-1 (MCP-1) production and activation of B cells, T cells and monocytes $[1,17,21,25,26]$. Recently, visfatin involvement was suggested in the pathogenesis of OA pain 
[18]. Little is known about resistin and OA [15]. One study reported a higher serum resistin concentration in OA patients compared with healthy controls, as well as a weak positive association between histological grade of synovial inflammation and resistin concentration [7].

The objectives of this exploratory study were to determine whether SF or serum adipokine concentrations are related to pain in patients with end-stage hip or knee OA, and whether adipokine concentrations and their relationship with pain might differ between joints.

\section{Materials and methods}

Study design and patient population

We conducted a cross-sectional study of patients with endstage hip or knee OA who presented for total joint arthroplasty (TJA) at a large orthopaedic centre between January and December 2010. Part of the following method's section has already been described in a previous publication on the same cohort [14]. Patients with secondary OA due to trauma or inflammatory arthritis were excluded. The study was approved by the local ethics committee and informed consent was obtained from all patients.

\section{Cytokine measurement}

SF (1 ml) was collected during surgery by the operating surgeon via direct aspiration through the joint capsule immediately after skin incision to minimise blood contamination. Because we only included elective TJA, they were all performed in the morning and early afternoon. Blood samples $(5 \mathrm{ml})$ were obtained in the morning during the pre-operative examination at the same time as standard pre-operative blood specimens were obtained. All blood and SF samples were immediately centrifuged. Serum and SF were aliquoted and frozen at $-80{ }^{\circ} \mathrm{C}$ until measurement. DuoSet enzyme-linked immunosorbent assay (ELISA) (R\&D Systems, Abingdon, UK) were used to determine IL-6, leptin, adiponectin and resistin concentrations according to the manufacturer. Minimum detectable cytokine concentrations for these assays was estimated to be $9.4 \mathrm{pg} / \mathrm{ml}$ for IL-6, $31.2 \mathrm{pg} / \mathrm{ml}$ for leptin, $62.5 \mathrm{pg} / \mathrm{ml}$ for adiponectin and $31.2 \mathrm{pg} / \mathrm{ml}$ for resistin. Both intra- and interassay coefficients of variation were $<10 \%$. Visfatin concentrations were measured using a Phoenix ELISA kit (Phoenix Pharmaceuticals, Inc., Burlingame, CA, USA) according to the manufacturer. The estimated minimum detectable visfatin concentration was $2.2 \mathrm{ng} / \mathrm{ml}$ (interassay variation $<15 \%$ and intraassay variation $<10 \%$ ). In addition, the adiponectin-leptin ratio was calculated to allow for comparison with a previous study [9].

\section{Outcome assessment}

The main outcome was the pre-operative pain level measured with the Western Ontario and McMaster Universities Osteoarthritis Index (WOMAC) pain score [2]. The score was analysed both as continuous $(0-100,100=$ no pain) and as dichotomised ( $>25$ vs. $\leq 25=$ quartile with greatest pain) variable; visual analog scale (VAS) for pain assessment evaluated as continuous variable $(0-10,0=$ no pain). Scores were sent to all patients seven to ten days prior to surgery. A medical secretary was involved in sending and collecting scores and questionnaires and in data entry. Baseline characteristics were obtained preoperatively at the entrance examination.

\section{Statistical analysis}

The patterns of adipokine distribution between synovial and blood compartments were studied according to OA site. The Mann-Whitney $U$ test was used to compare concentrations between hip and knee OA groups, and Spearman's correlation coefficients were calculated to assess correlations between the two compartments. The association between SF concentrations and pain was evaluated as continuous variable using Spearman's correlation coefficient and as categorical variable using quartiles. Relative risks (RR) and their $95 \%$ confidence intervals (CI) were calculated to compare proportions of patients with the highest pain level among those in the highest quartile of adipokine concentration vs. first to third quartiles. Logistic regression analysis with WOMAC pain dichotomised as dependent variable was used to adjust for sex, body mass index (BMI) and smoking.

\section{Results}

A total of 206 patients with complete SF analysis and pain evaluation were assessed: 112 with hip and 94 with knee OA. The group with hip OA comprised 58 women $(51.8 \%)$ with a median age of 72 years [interquartile range (IQR) 66-78) and a median BMI $27 \mathrm{~kg} / \mathrm{m}^{2}$ (IQR 24-29.6); $23 \%$ were obese. Regarding medical comorbidities: $39.3 \%$ were ever-smokers, $9.8 \%$ had diabetes, $61.6 \%$ had hypertension and $8 \%$ had cardiac disease. The group with knee OA comprised 64 women (68.1\%), median age was 74.5 years (IQR 68-79.3) and median BMI was $28.3 \mathrm{~kg} / \mathrm{m}^{2}$ (IQR 25.4-31.2); $33 \%$ were obese. Regarding medical comorbidities, $26.1 \%$ were eversmokers, $13.8 \%$ had diabetes, $64.9 \%$ had hypertension and $4.3 \%$ had cardiac disease. Of the 206 patients, 186 (90.3\%) also had serum analyses available: 109 with hip and 77 with knee OA. 


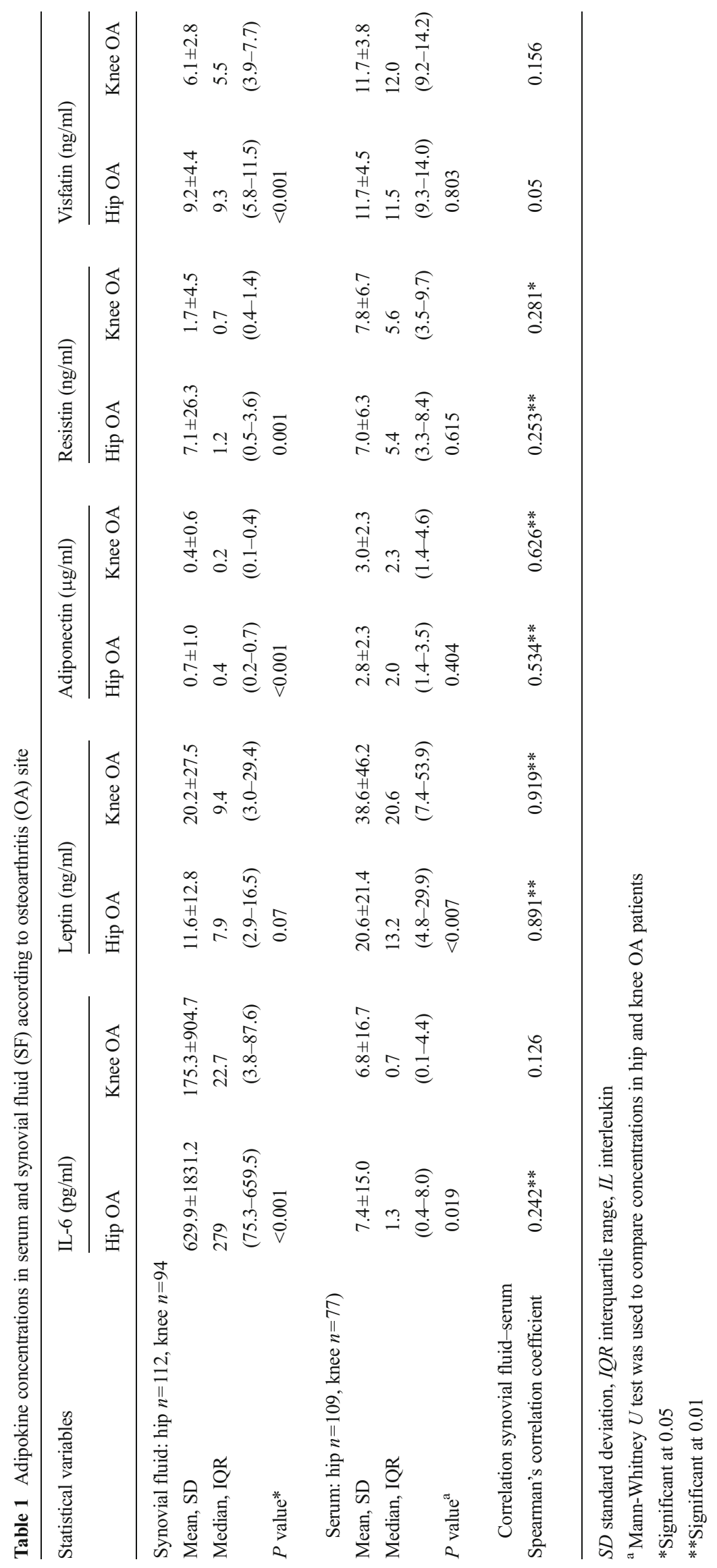


Adipokine concentrations according to OA site

All adipokine levels were significantly higher in the SF of hip joints than in the SF of knee joints, except for leptin, which tended to be higher in the knee (Table 1). In serum, hip OA patients had significantly higher IL-6 concentrations, and knee OA patients had significantly higher leptin concentrations; the level of the other adipokines did not differ according to OA site. Median serum concentrations of leptin, adiponectin, resistin and visfatin exceeded those in SF, whereas for IL-6, median concentrations were much higher in SF (hip 279.0 vs. $1.3 \mathrm{pg} / \mathrm{ml}$; knee 22.7 vs. $0.7 \mathrm{pg} / \mathrm{ml}$ ).

Correlation between serum and SF adipokine concentrations

Leptin and adiponectin concentrations were highly correlated in serum and SF both in hip and knee OA (Table 1). There was a weak correlation for resistin, and no significant correlation was observed for visfatin. Serum and SF IL-6 concentrations were significantly (weakly) correlated only in patients with hip OA.

Association between SF adipokine concentrations and pre-operative pain

In patients with hip OA, worse pain scores were significantly correlated with increased SF IL-6 and visfatin concentrations (Table 2). In contrast, in patients with knee OA, worse pain scores were significantly correlated with increased SF leptin concentrations and decreased SF adiponectin-leptin ratio. In serum, only SF leptin concentrations in the knee OA group were linearly correlated with pain.

In hip OA, patients with the highest degree of pain (WOMAC score $\leq 25$ ) had more often significantly higher concentrations of SF IL-6 (fourth quartile vs. first to third: RR 2.2; $95 \%$ CI 1.2-3.8), visfatin (RR 2.4; $95 \%$ CI 1.4-4.2) and leptin (RR 2.4; 95 \% CI 1.5-4.0), (Fig. 1). In knee OA, patients with the highest degree of pain had more often significantly high SF leptin (RR 2.7; 95 \% CI 1.3-5.8) concentrations and linearly decreased SF adiponectin concentrations (in quartiles, $p$ for trend 0.013). The relationship remained significant for SF visfatin and leptin in hip and for SF leptin in knee OA after adjusting for BMI, sex and smoking.

\section{Discussion}

Our findings support a differential relationship between intraarticular concentrations of several adipokines and preoperative pain for hip and knee OA. They further demonstrate differential adipokine levels by joints, with higher levels of SF IL-6, adiponectin, resistin and visfatin in hip than in knee $\mathrm{OA}$, and higher SF leptin concentration in knee OA compared with hip OA. The observed differences are in agreement with the established notion that hip OA stands out as a different

Table 2 Correlation between synovial fluid (SF) adipokine concentrations, body mass index (BMI) and pain

\begin{tabular}{|c|c|c|c|c|c|c|c|}
\hline & Leptin & Adiponectin & Resistin & Visfatin & BMI & VAS pain & WOMAC pain \\
\hline \multicolumn{8}{|l|}{ Hip $(n=112)$} \\
\hline IL-6 & $0.342 * *$ & 0.095 & -0.038 & $0.296 * *$ & 0.082 & $0.275^{* *}$ & $-0.338 * *$ \\
\hline Leptin & & 0.084 & -0.021 & 0.139 & $0.435 * *$ & 0.116 & -0.163 \\
\hline Adiponectin & & & $0.235^{*}$ & $0.258 * *$ & -0.051 & 0.159 & -0.116 \\
\hline Adiponectin-leptin ratio & & & & & $-0.335^{* *}$ & 0.034 & 0.026 \\
\hline Resistin & & & & $0.297 * *$ & 0.102 & 0.001 & 0.062 \\
\hline Visfatin & & & & & 0.073 & $0.242 *$ & $-0.279 * *$ \\
\hline \multicolumn{8}{|l|}{ Knee $(n=94)$} \\
\hline IL-6 & 0.124 & 0.14 & 0.071 & -0.052 & -0.093 & 0.057 & 0.032 \\
\hline Leptin & & $0.208 *$ & 0.056 & 0.153 & $0.575^{* *}$ & 0.194 & $-0.258 *$ \\
\hline Adiponectin & & & 0.153 & $0.220 *$ & 0.056 & -0.096 & 0.184 \\
\hline Adiponectin-leptin ratio & & & & & $-0.414 * *$ & -0.195 & $0.293 * *$ \\
\hline Resistin & & & & 0.123 & 0.093 & -0.096 & 0.047 \\
\hline Visfatin & & & & & 0.16 & 0.101 & 0.054 \\
\hline
\end{tabular}

Spearman's correlation coefficients

IL interleukin, VAS visual analogue scale, WOMAC Western Ontario and McMaster Universities Osteoarthritis Index

*Significant at 0.05

**Significant at 0.01 


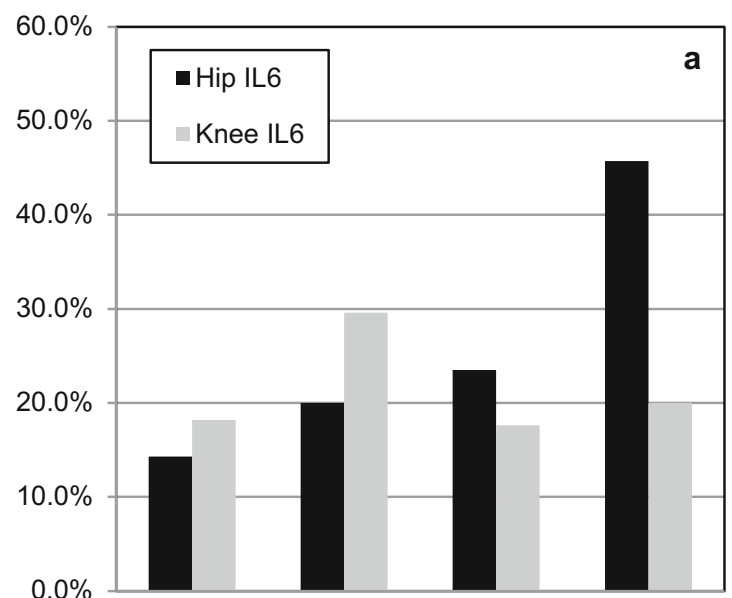

1st quartile 2 nd quartile 3 rd quartile 4 th quartile Intra-articular IL6 concentration
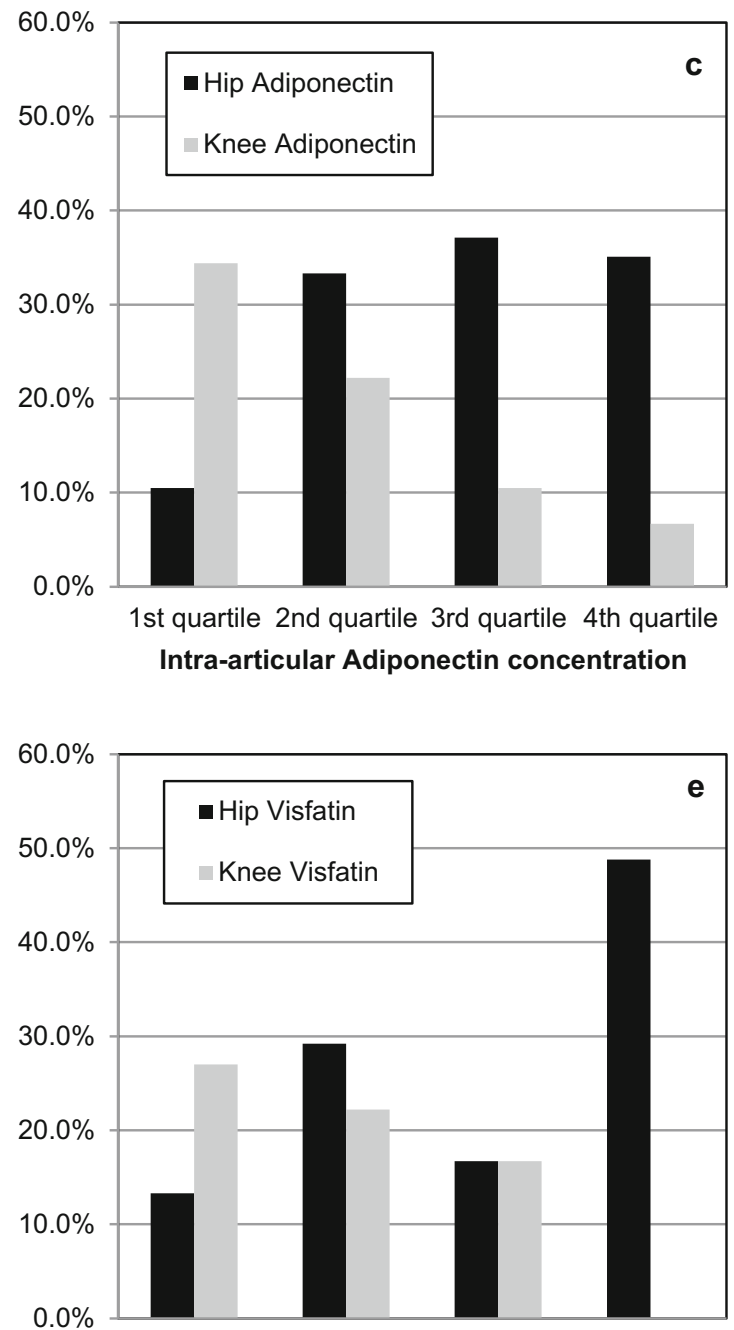

1st quartile 2 nd quartile 3 rd quartile 4 th quartile Intra-articular Visfatin concentration
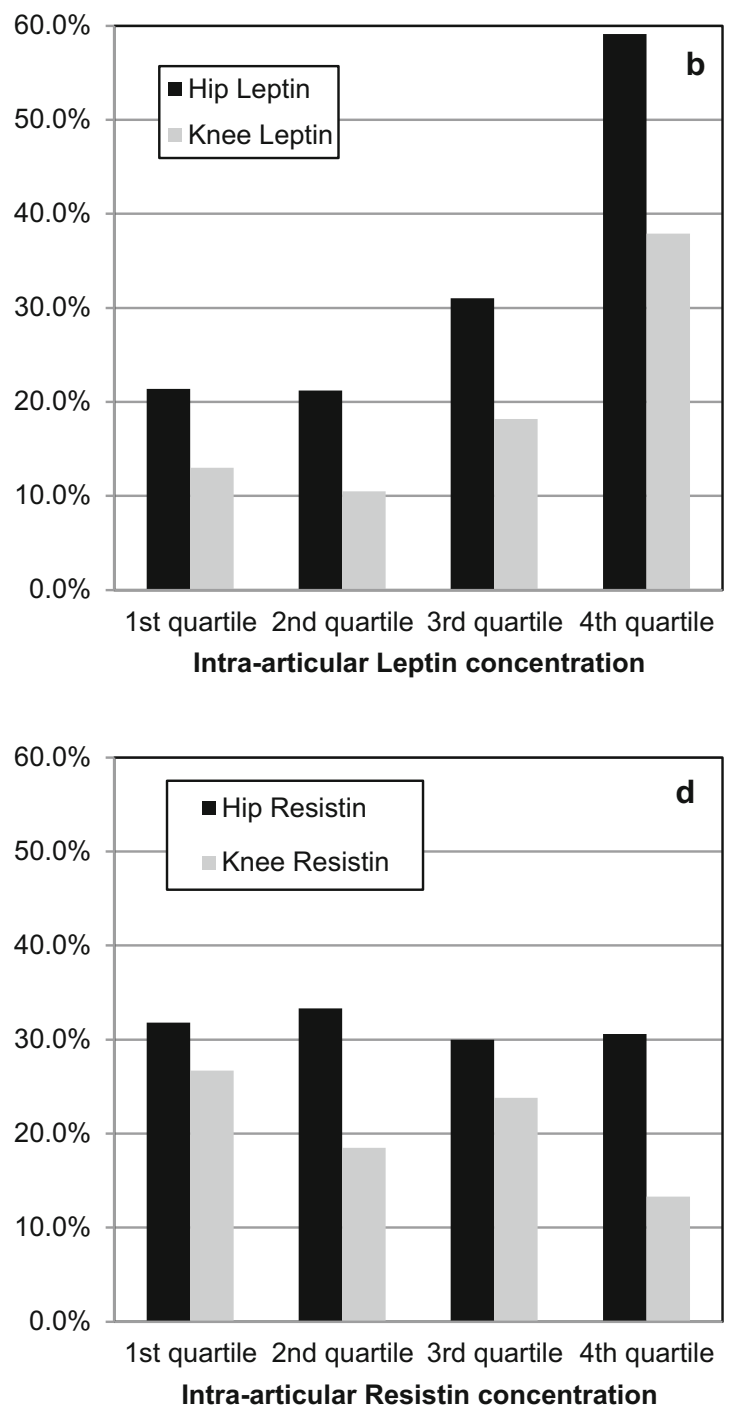

Fig. 1 a-e Proportion with highest Western Ontario McMaster Universities Osteoarthritis Index (WOMAC) pain level ( $\leq 25)$ according to quartiles of synovial fluid (SF) adipokine concentrations 
entity. It often occurs alone, at a younger age, in a higher proportion of men and with a different set of risk factors [6].

The associations of SF IL-6 and visfatin with pain in hip OA have not been reported so far. Our results corroborate previous clinical studies reporting an association between IL-6 levels and chronic low back pain [12] and between visfatin levels and pain in incipient upper-extremity soft-tissue disorders [20]. Interestingly, a recent experimental study suggested involvement of visfatin in the pathogenesis of OA pain via induction of nerve growth factor (NGF) release [18]. Other experimental studies using human adipocytes [25] and human endothelial cells [1] showed that visfatin stimulated in a dosedependent fashion the production of MCP-1, a chemokine regulating migration and infiltration of monocytes/ macrophages, which has recently been reported to mediate pain in experimental OA [16]. Regarding IL-6 and its influence on pain, an experimental study on rats [4] found an association between IL- 6 and increased mechanical hypersensitivity, possibly through an action of the cytokine on nerve fibres. SF IL-6 and visfatin concentrations were correlated in hip OA patients in our study, confirming the findings of Moschen et al. of a strong correlation between visfatin and markers of inflammation, such as IL-6 [17].

We previously demonstrated a positive association between SF leptin and increased pain in patients with hip and knee OA [14]. Moreover, in knee OA patients, adiponectin and adiponectin-leptin ratio were correlated with pain, a finding in accordance with Gandhi et al. [9]. No correlation between resistin and OA pain in hip or knee was found, a finding also reported by Massengale et al. in their study on adipokines and pain in hand OA [15].

So far, clinical studies on the relationship between adipokines and pain in OA are limited by small samples, no stratification of adipokine concentrations by OA joints or a limited number of adipokines examined. The study reported here involved a large number of patients for whom concentrations of five different adipokines were determined in hip and knee SF and serum, all at the same time. Nevertheless, our study is limited by its cross-sectional design, precluding evaluation of causal relationships. Moreover, adipokines show diurnal variations [3, 24], and we cannot exclude that this factor may have partly influenced our results regarding SF concentrations, which were obtained during a time span ranging from morning to early afternoon.

In conclusion, our findings support a connection between intra-articular concentrations of several adipokines and severity of preoperative pain, which differs between patients with hip and knee OA. In hip OA, pain was associated with IL-6 and visfatin. In knee $\mathrm{OA}$, pain was associated with adiponectin, whereas leptin played a role in both hips and knees. These results may help further characterise different OA phenotypes and better target pain treatment.
Acknowledgments The authors express their deep appreciation to all orthopaedic surgeons, operating-room personnel and especially to Mme Carole Bandi and Mme Madeleine Vuillet for their efforts in data collection.

Contributors AL, SB, AF and CG participated in the study design. AL conducted the statistical analysis. SB, GJP, DS and $\mathrm{PH}$ participated in data collection. All authors participated in data interpretation and drafting of the manuscript.

Funding statement and competing interests All authors have completed the Unified Competing Interest form at www.icmje.org/coi disclosure.pdf (available on request from the corresponding author) and declare: Institutional financial support was obtained from the "Fondation pour la recherche ostéo-articulaire" and internal institutional funding from the Geneva University Hospitals was received for this study. The funding sources had no role in data collection, analysis or interpretation, in preparation of the manuscript or in its submission for publication. There were no other financial relationships with any organisations that might have an interest in the submitted work in the previous 3 years and no other relationships or activities that could appear to have influenced the submitted work.

Ethical approval Ethical approval for the study No CER 09-215 (09072) was obtained from the Ethical Committee of the Geneva University Hospitals.

\section{References}

1. Adya R, Tan BK, Chen J, Randeva HS (2009) Pre-B cell colony enhancing factor (PBEF)/visfatin induces secretion of MCP-1 in human endothelial cells: Role in visfatin-induced angiogenesis. Artherosclerosis 205:113-119

2. Bellamy N, Buchanan WW, Goldsmith CH, Campbell J, Stitt LW (1988) Validation study of WOMAC: a health status instrument for measuring clinically important patient relevant outcomes to antirheumatic drug therapy in patients with osteoarthritis of the hip or knee. $\mathrm{J}$ Rheumatol 158:1833-1840

3. Benedict C, Shostak A, Lange T, Brooks SJ, Schiöth HB, Schultes B, Born J, Oster H, Hallschmid M (2012) Diurnal rhythm of circulating nicotinamide phosphoribosyltransferase (Nampt/visfatin/PBEF): impact of sleep loss and relation to glucose metabolism. J Clin Endocrinol Metab 97:E218-222

4. Brenn D, Richter F, Schaible HG (2007) Sensitization of unmyelinated sensory fibres of the joint nerve to mechanical stimuli by interleukin-6 in the rat. An inflammatory mechanism of joint pain. Arthritis Rheum 56:351-359

5. Chen WP, Bao JP, Feng J, Hu PF, Shi ZL, Wu LD (2010) Increased serum concentrations of visfatin and its production by different joint tissues in patients with osteoarthritis. Clin Chem Lab Med 48:11411145

6. Cushnaghan J, Dieppe P (1991) Study of 500 patients with limb joint osteoarthritis. I. Analysis by age, sex, and distribution of symptomatic joint sites. Ann Rheum Dis 50:8-13

7. de Boer TN, van Spil WE, Huisman AM, Polak AA, Bijlsma JW, Lafeber FP, Mastbergen SC (2012) Serum adipokines in osteoarthritis; comparison with controls and relationship with local parameters of synovial inflammation and cartilage damage. Osteoarthritis Cartilage 20:846-853

8. Doss F, Menard J, Hauschild M, Kreutzer HJ, Mittlmeier T, MullerSteinhardt M, Muller B (2007) Elevated IL-6 levels in the synovial fluid of osteoarthritis patients stem from plasma cells. Scand J Rheumatol 36:136-139 
9. Gandhi R, Takahashi M, Smith H, Rizek R, Mahomed NN (2010) The synovial fluid adiponectin-leptin ratio predicts pain with knee osteoarthritis. Clin Rheumatol 29:1223-1228

10. Gomez R, Conde J, Scotece M, Gomez-Reino JJ, Lago F, Gualillo O (2011) What's new in our understanding of the role of adipokines in rheumatic diseases? Nat Rev Rheumatol 7:528-536

11. Gosset M, Berenbaum F, Salvat C, Sautet A, Pigenet A, Tahiri K, Jacques C (2008) Crucial role of visfatin/pre-B cell colony-enhancing factor in matrix degradation and prostaglandin E2 synthesis in chondrocytes: possible influence on osteoarthritis. Arthritis Rheum 58:1399-409

12. Heffner KL, France CR, Trost Z, Ng HM, Pigeon WR (2011) Chronic low back pain, sleep disturbance, and interleukin-6. Clin J Pain 27:35-41

13. Kotake S, Sato K, Kim KJ, Takahashi N, Udagawa N, Nakamura I, Yamaguchi A, Kishimoto T, Suda T, Kashiwazaki S (1996) Interleukin-6 and soluble interleukin-6 receptors in the synovial fluids from rheumatoid arthritis patients are responsible for osteoclast-like cell formation. J Bone Miner Res 11:88-95

14. Lubbeke A, Finckh A, Puskas GJ, Suva D, Ladermann A, Bas S, Fritschy D, Gabay C, Hoffmeyer P (2013) Do synovial leptin levels correlate with pain in end-stage arthritis? Int Orthop 37:2071-2079

15. Massengale M, Lu B, Pan JJ, Katz JN, Solomon DH (2012) Adipokine hormones and hand osteoarthritis: radiographic severity and pain. PLoS One 7:e47860

16. Miller RE, Tran PB, Das R, Ghoreishi-Haack N, Ren D, Miller RJ, Malfait AM (2012) CCR2 chemokine receptor signaling mediates pain in experimental osteoarthritis. Proc Natl Acad Sci U S A 109: 20602-20607

17. Moschen AR, Kaser A, Enrich B, Mosheimer B, Theurl M, Niederegger H, Tilg H (2007) Visfatin, an adipocytokine with proinflammatory and immunomodulating properties. J Immunol 178: 1748-1758

18. Pecchi E, Priam S, Gosset M, Pigenet A, Sudre L, Laiguillon MC, Berenbaum F, Houard X (2014) Induction of nerve growth factor expression and release by mechanical and inflammatory stimuli in chondrocytes: possible involvement in osteoarthritis pain. Arthritis Res Ther 16:R16 [Epub ahead of print]

19. Presle N, Pottie P, Dumond H, Guillaume C, Lapicque F, Pallu S, Mainard D, Netter P, Terlain B (2006) Differential distribution of adipokines between serum and synovial fluid in patients with osteoarthritis. Contribution of joint tissues to their articular production. Osteoarthritis Cartilage 14: 690-695

20. Rechardt M, Shiri R, Lindholm H, Karppinen J, Viikari-Juntura E (2013) Associations of metabolic factors and adipokines with pain in incipient upper extremity soft tissue disorders: a cross-sectional study. BMJ Open 3:e003036

21. Rongvaux A, Galli M, Denanglaire S, Van Gool F, Drèze PL, Szpirer C, Bureau F, Andris F, Leo O (2008) Nicotinamide phosphoribosyl transferase/pre-B cell colony-enhancing factor/visfatin is required for lymphocyte development and cellular resistance to genotoxic stress. J Immunol 181:4685-95

22. Schaffler A, Ehling A, Neumann E, Herfarth H, Tarner I, Scholmerich J, Muller-Ladner U, Gay S (2003) Adipocytokines in synovial fluid. Jama 290:1709-1710

23. Sellam J, Berenbaum F (2013) Is osteoarthritis a metabolic disease? Joint Bone Spine 80:568-573

24. Sinha MK, Ohannesian JP, Heiman ML, Kriauciunas A, Stephens TW, Magosin S, Marco C, Caro JF (1996) Nocturnal rise of leptin in lean, obese, and non-insulin-dependent diabetes mellitus subjects. J Clin Invest 97:1344-1347

25. Sommer G, Kralisch S, Kloting N, Kamprad M, Schrock K, Kratzsch J, Tonjes A, Lossner U, Bluher M, Stumvoll M, Fasshauer M (2010) Visfatin is a positive regulator of MCP-1 in human adipocytes in vitro and in mice in vivo. Obesity 18:1486-1492

26. Sun Z, Lei H, Zhang Z (2013) Pre-B cell colony enhancing factor (PBEF), a cytokine with multiple physiological functions: Cytokine Growth Factor Rev 24:433-442

27. Tilg H, Moschen AR (2006) Adipocytokines: mediators linking adipose tissue, inflammation and immunity. Nat Rev Immunol 6: $772-783$ 